\title{
Electronic and structural transition in $\mathrm{La}_{0.2} \mathrm{Sr}_{0.8} \mathrm{MnO}_{3}$
}

\author{
R. Bindu and Kalobaran Maiti \\ Department of Condensed Matter Physics and Materials Science, \\ Tata Institute of Fundamental Research, Homi Bhabha Road, Colaba, Mumbai - 400 005, INDIA. \\ R. Rawat \\ UGC-DAE Consortium for Scientific Research, University Campus, Khandwa Road, Indore 452 017, India.
}

\author{
S. Khalid \\ National Synchrotron Light Source, Brookhaven National Laboratory, Upton, New York - 11973
}

(Dated: October 27, 2018)

\begin{abstract}
We investigate the interplay of the electronic and structural transition in $\mathrm{La}_{0.2} \mathrm{Sr}_{0.8} \mathrm{MnO}_{3}$. The transport and specific heat measurements exhibit unusual evolutions and signature of a first order phase transition around $265 \mathrm{~K}$. Mn K-edge extended $x$-ray absorption fine structure results reveal distortion in the $\mathrm{MnO}_{6}$ octahedra even in the cubic phase and a remarkable evolution of the distortion across the phase transition. These results manifest the importance of fluctuations in Mn $3 d$ orbital occupancy and disorder in their electronic properties, which may help in understanding the orbital and spin ordering proposed in these systems.

PACS numbers: $61.50 . \mathrm{Ks}, 75.30 . \mathrm{Kz}, 75.47 . \mathrm{Lx}, 61.05 \mathrm{cj}$
\end{abstract}

Discovery of colossal magnetoresistance (CMR) in manganites have opened up a new field of research in solid state physics due to their interesting fundamental issues and significant technological applications such as high density storage media, field sensors, etc. These systems exhibit plethora of exotic properties such as charge, orbital and spin ordering, electronic phase separation, etc. in addition to the CMR effect. It is now realized that the intricate competition between the charge, spin, orbital, and lattice degrees of freedom in these mixed valent manganites are important for these exotic properties $\frac{1,2,3}{2}$

Among all the manganites, $\mathrm{La}_{1-x} \mathrm{Sr}_{x} \mathrm{MnO}_{3}(x<0.5)$ is studied the most due to its proximity to the CMR effect. With the increase in $x$, the tolerance factor becomes close to unity and the Mn-O-Mn angle also becomes close to $180^{\circ}$. In this situation, $\mathrm{Mn} e_{g}$ orbitals would be almost degenerate ${ }^{4,5}$ and is ideal to study the electronic charge distribution in Mn $3 d$ levels across the electronic phase transitions observed in these systems.

In this paper, we report our results on the electronic and structural evolution associated to $\mathrm{MnO}_{6}$ octahedra in $\mathrm{La}_{0.2} \mathrm{Sr}_{0.8} \mathrm{MnO}_{3}$ as a function of temperature. This compound exhibits a transition from paramagnetic to C-type antiferromagnetic (C-AFM) structure around $265 \mathrm{~K} . \underline{6}$ Reports on structural transition are controversial suggesting a cubic to tetragona $\underline{1}^{\underline{6}}$ or no structural transition ${ }^{7}$ with temperature. Subsequent theoretical studies 4,5 predict importance of orbital degeneracy in this system. The orbital degeneracy of Mn $3 d$ states strongly depends on the local crystal structure of the $\mathrm{MnO}_{6}$ octahedra, which can be probed efficiently by extended $x$-ray absorption fine structure (EXAFS) technique. Thus, we have employed Mn K-edge EXAFS around the region of electronic phase transition to investigate the evolution of the local structural parameters of the $\mathrm{MnO}_{6}$ octahedra. Our results reveal that the $\mathrm{MnO}_{6}$ octahedra are distorted even in the cubic phase. The results at lower temperatures manifest phenomenal evolution of the Mn-O bond lengths across the phase transition.

The sample was prepared by conventional solid state route ${ }^{8}$ and characterized by powder $x$-ray diffraction technique. Sample was found to be in single phase with no signature of impurity feature. The analysis of the diffraction peaks at room temperature indicates cubic structure with space group $P m \overline{3} m$. Iodometric redox titration confirmed the sample to be stoichiometric. The resistivity and heat capacity measurements were performed using standard four probe method and home made semi adiabatic heat pulse calorimeter, respectively. Mn K-edge EXAFS studies were carried out at X-18B beamline at the National Synchrotron Light Source, Brookhaven National Laboratory with an eneregy resolution of $0.8 \mathrm{eV}$ at $\mathrm{Mn} \mathrm{K}$-edge.

EXAFS analysis was carried out using UWXAS software $e^{\underline{\underline{a}}}$ following the standard procedure $\underline{\underline{10}}$ The Fourier transform (FT) to the $R$-space was carried out in the $k$-range $3-13 \AA^{-1}$ by Fourier transforming $k^{2} \chi(k)$ $(\chi(k)$ is the EXAFS oscillations) by a Hanning window function. The back scattering amplitude and phases were calculated using FEFF6.01a code ${ }^{11}$ for $\mathrm{LaMnO}_{3}$ and the same were used for all the temperatures. The first coordination shell fit was done in the Fourier filtered $k$-space in the range $0.86-1.87 \AA$. The overall many body reduction factor, $S_{0}^{2}$ was fixed to 0.82 . As seen earlier ${ }^{12}$, we find that the Mn K-edge EXAFS data at room temperature are best described by 2 short $\mathrm{Mn}-\mathrm{O}$ bond lengths, $R_{1}$ and 4 long Mn-O bond lengths, $R_{2}$. In order to avoid uncertainty in the fitting procedure, we have considered average bond distances for eight $\mathrm{Mn}-\mathrm{Sr} / \mathrm{La}$ bonds and six Mn-Mn bonds in higher coordination shells (0.86 - 3.87 $\AA)$.

The temperature dependent resistivity, $\rho$ and heat ca- 
pacity, $C_{p}$ are plotted in Fig. 1. $\rho$ in Fig. 1(a) exhibits insulating temperature dependence along with a sharp increase at about $265 \mathrm{~K}\left(T_{1}\right)$ and a change of slope at about $254 \mathrm{~K}$. In addition, a distinct hysteresis is observed around $T_{1}$ and $224 \mathrm{~K}\left(T_{2}\right)$ for the cooling and warming cycles. This is demonstrated in Fig. 1(b), where we show the difference in $\rho$ between cooling $\left(\rho_{c}\right)$ and warming $\left(\rho_{w}\right)$ cycles normalized by $\rho_{c}$. It is evident that $\left(\rho_{c}-\rho_{w}\right) / \rho_{c}$ exhibits two peaks of opposite signs at around $T_{1}$ and $T_{2}$. The change in sign suggests significant modification in transport mechanisms at different temperatures. The hysteresis observed in resistivity is an indication of first order phase transition. Notably, the transition from paramagnetic to $\mathrm{C}$-type antiferromagnetic phase ${ }^{\underline{6}}$ is also observed at $T_{1}$.

The heat capacity data shown in Fig. 1(c) exhibit a sharp peak at $T_{1}$. The magnetic entropy change at this transition obtained by subtracting the polynomial background is found to be very large, $4.3 \mathrm{~J} / \mathrm{mol} . \mathrm{K}$. No distinct feature is observed around $T_{2}$. Interestingly, at low temperatures, $C_{p} / T$ exhibit a linear dependence with $T^{2}$ as shown in Fig. 1(d). Considering $C_{p} / T=\gamma+\beta T^{2}$, the value of $\gamma$ is found to be $5.6 \mathrm{~mJ} / \mathrm{mol}^{2} \mathrm{~K}^{2}$. In metals, $\gamma$ corresponds to the electronic contribution to $C_{p}$. However, the compound in this study exhibits insulating temperature dependence. Thus, the observation of large $\gamma$ indicating finite density of states at the Fermi level is unusual and needs further study to understand this effect.

In Fig. 2(a), we show the temperature evolution of the modulus of FT $\left[\mathrm{k}^{2} \chi(\mathrm{k})\right]$. The FT curves are uncorrected for the central and back scattering phase shifts. The figure shows three prominent peaks around 1.5, 2.9 and 3.4 $\AA$ A marked by A, B and C, respectively. Peaks A and B correspond to $\mathrm{Mn}-\mathrm{O}$ and $\mathrm{Mn}-\mathrm{Sr} / \mathrm{La}$ shells, respectively, and peak $\mathrm{C}$ corresponds to $\mathrm{Mn}-\mathrm{Mn}$ and multiple scattering paths (Mn-O-Mn). While there is no apparent shift in the peak position of the peaks $\mathrm{B}$ and $\mathrm{C}$, their relative intensity decreases with the decrease in temperature as shown by plotting the maxima of $\mathrm{B}$ and $\mathrm{C}$ in Fig. 2(b). At $T>270 \mathrm{~K}$, the peak B is weaker than peak C. For $270 \mathrm{~K}>T>230 \mathrm{~K}$, the intensities are very similar and at $T<230 \mathrm{~K}$, the intensity of peak B increases significantly relative to the intensity of peak C. Peak A exhibits a small shift towards the origin and an increasing trend in intensity with the decrease in temperature. Since, $\chi(k)$ is a function of bond length, $R_{j}$ and Debye-Waller factor, $\sigma_{j}$ for the $j$-th coordination shell, such modification in FT amplitude indicates significant changes in these two parameters with temperatures. In order to obtain the detailed structural information of these modifications, we have carried out the EXAFS fitting on Mn-O, Mn-Sr/La and $\mathrm{Mn}-\mathrm{Mn}$ coordination shells. Representative fit of these shells are shown in Fig. 2(c) and 2(d). The typical $\mathrm{R}$-factors for the first and higher coordination shell fits are 0.003 and 0.015 , respectively.

First, we start with the first coordination shell corresponding to Mn-O bonds. This shell is fitted with 2 short bonds, $R_{1}$ and 4 long bonds, $R_{2}$ and the results are shown in Fig. 3(a). It is evident from the figure that the distribution in Mn-O bonds persists with the decrease in temperature until $270 \mathrm{~K}$. Interestingly, around the region of phase transition $(\sim 265 \mathrm{~K}), \mathrm{R}_{1}$ and $\mathrm{R}_{2}$ are almost identical indicating an undistorted $\mathrm{MnO}_{6}$ octahedron. At low temperatures, $R_{1}$ becomes significantly smaller than $R_{2}$ thereby $\mathrm{MnO}_{6}$ octahedra become distorted. This is remarkable and reveals presumably for the first time such behavior as a function of temperature across a first order phase transition. This suggests that tetragonal distortion starts from a perfectly cubic structure, where the $\mathrm{MnO}_{6}$ octahedra are also symmetric. A magnetic phase transition (from PM to C-AFM) also occurs at $T_{1}$. Interestingly, in sharp contrast to the common belief, the Mn-atoms corresponding to the shorter bond length, $R_{1}$ becomes ferromagnetically coupled and the ones with the longer bond lengths, $R_{2}$ are antiferromagnetically coupled.

When the $\mathrm{MnO}_{6}$ octahedra are symmetric, the $d_{x y}$, $d_{y z}$ and $d_{x z}$ orbitals are degenerate forming a $t_{2 g}$ band, and $d_{x^{2}-y^{2}}$ and $d_{z^{2}}$ orbitals are degenerate forming an $e_{g}$ band. In the ionic configuration, Mn $3 d$ bands in $\mathrm{La}_{0.2} \mathrm{Sr}_{0.8} \mathrm{MnO}_{3}$ have 3.2 electrons and the up spin $t_{2 g}$ band will be completely filled. The structural changes in the $\mathrm{MnO}_{6}$ octahedra will lead to a change in the degeneracy of the $t_{2 g}$ and $e_{g}$ bands, and hence significant fluctuation in occupancy of $d_{x^{2}-y^{2}}$ and $d_{z^{2}}$ orbitals. Since, the octahedra are compressed along apical direction $\left(R_{1}\right.$; say $z$-direction), the $d$ orbital corresponding to the plane having larger Mn-O bond lengths $\left(R_{2} ; d_{x^{2}-y^{2}}\right)$ will be more populated compared to the perpendicular one. This is presumably the reason for the anomalous magnetic coupling observed in this system as filled up spin $d_{x^{2}-y^{2}}$ band favors antiferromagnetic coupling due to superexchange interaction.

In order to explore plethora of unusual effects such as the sharp peak in $C_{p}$, a steep increase in resistivity and the nucleation of unusual magnetic coupling at $T_{1}$ further, we show the Debye-Waller factors corresponding to $R_{1}$ and $R_{2}$ in Fig. 3(d) and 3(f). The Debye-Waller factor corresponding to $R_{1}$ shown in Fig. 3(d) is significantly large. This suggests strong influence of disorder in localizing the Mn-O hybridized electronic states corresponding to $R_{1}$. This is presumably the reason for insulating transport despite the fact that ferromagnetic coupling along $R_{1}$ often favors metallic conduction and finite $\gamma$ corresponds to finite density of states at the Fermi level.

In Figs. 3(b) and 3(c), we show the Mn-Sr/La and MnMn bond lengths, respectively and corresponding DebyeWaller factors are shown in Fig. 3(e) and 3(f), respectively. It is evident from the figures that around $\mathrm{T}_{1}$, the average $\mathrm{Mn}-\mathrm{Sr} / \mathrm{La}$ and $\mathrm{Mn}-\mathrm{Mn}$ bond lengths remain almost the same.

At $245 \mathrm{~K}, \mathrm{MnO}_{6}$ octahedra are the most distorted ones and the Mn-Mn distance also becomes minimum. Below this temperature, the distortion in $\mathrm{MnO}_{6}$ octahedra re- 
duces gradually with the decrease in temperature and subsequently, the Mn-Mn distance also increases gradually. The Debye-Waller factor corresponding Mn-Mn distance does not change significantly (see Fig 3(f)) and that corresponding to $\mathrm{Sr} / \mathrm{La}$ atoms around $\mathrm{Mn}$ shown in Fig. 3(e) exhibits a gradual decrease with temperature. This indicates that the disorder in Sr-sublattice may not have significant role in the electronic properties of this compound.

Around $\mathrm{T}_{1}$, the value of resistivity during warming cycle $\left(\rho_{w}\right)$ is more than the resistivity during cooling cycle $\left(\rho_{c}\right)$. Below $257 \mathrm{~K}$, the value of $\rho_{c}$ becomes more than $\rho_{w}$ and a change in slope is also observed. Such unusual modification in transport behavior presumably due to the temperature induced structural evolution of the $\mathrm{MnO}_{6}$ octahedra leading to fluctuation of the occupancy of the Mn $e_{g}$ orbitals in the presence of strong disorder.
In summary, we have studied the electronic properties of $\mathrm{La}_{0.2} \mathrm{Sr}_{0.8} \mathrm{MnO}_{3}$ using resistivity and heat capacity measurements. Transport and heat capacity data reveal a first order phase transition around $265 \mathrm{~K}$. The local structure was probed by Mn K-edge EXAFS technique. The $\mathrm{MnO}_{6}$ octahedra are found to be distorted even in the cubic phase at room temperature. EXAFS results at different temperatures manifests remarkable evolution of the $\mathrm{MnO}_{6}$ octahedra across the first order phase transition. We observe that the tetragonal distortion and the antiferromagnetic ordering nucleates from a perfectly cubic structure, where the $\mathrm{MnO}_{6}$ octahedra are symmetric. Thus, the phase transition in this compound involves significant fluctuation in the Mn $3 d$ orbital occupancy and disorder induced localization, which may be important in understanding interesting orbital and spin ordering observed in manganites.
1 J. M. D. Coey, M. Viret and S. von Molnár, Adv. Phys. 48, 167 (1999).

2 M. B. Salamon and M. Jaime, Rev. Mod. Phys. 73, 583 (2001).

3 E. Dagotto, T. Hotta, A. Moreo, Physics Reports. 344, 1 (2001).

4 J. van den Brink, G. Khaliullin, and D. Khomskii, Phys. Rev. Lett. 83, 5118 (1999).

5 R. Maezono, S. Ishihara and N. Nagaosa, Phys. Rev. B. 58, 11583 (1998).

6 O. Chmaissem, B. Dabrowski, S. Kolesnik, J. Mais, J. D. Jorgensen and S. Short, Phys. Rev. B 67, 094431 (2003).

7 J. Hemberger, A. Krimmel, T. Kurz, H. -A. Krug von Nidda, V. Yu. Ivanov, A. A. Mukhin, A. M. Balbashov, and A. Loidl, Phys. Rev. B. 66, 094410 (2002).

${ }^{8}$ R. Bindu, Eur. Phys. J. B. 37, 321 (2004).

9 E. A. Stern, M. Newville, B. Ravel, Y. Yacoby, D. Haskel, Physica B. 208, 117 (1995).

$10 X$-ray absorption: Principles, Applications, Techniques of EXAFS, SEXAFS and XANES, edited by D. C. Konningsberger and R. Prins (John Wiley and Sons, New York, 1988).

11 S. I. Zabinsky, J. J. Rehr, A. Ankudinov, R. C. Albers and M. J. Eller, Phys. Rev. B. 52, 2995 (1995).

12 R. Bindu, S. K. Pandey, A. Kumar, S. Khalid and A. V.
Pimpale, J. Phys: Cond. Matt. 17, 6393 (2005).

\section{FIGURE CAPTIONS:}

Fig. 1: (color online) (a) The resistivity during warming (open circles), cooling (line) cycles. (b) The difference in the resistivity during the cooling $\left(\rho_{c}\right)$ and warming $\left(\rho_{w}\right)$ cycles normalized by $\rho_{c}$. (c) The heat capacity, $C_{p}$ as a function of temperature. (d) $C_{p} / T$ vs $T^{2}$ ( $T=$ temperature) shown in the low temperature region.

Fig. 2: (color online) (a) The Fourier transform (FT) of $k^{2} \chi(k)$ at different temperatures. (b) The intensities of the peaks $\mathrm{A}, \mathrm{B}$ and $\mathrm{C}$ as a function of temperature. Representative fits for the case of (c) first and (d) higher coordination shells at $255 \mathrm{~K}$. The open circles and the solid line indicate the experimental and the fitted patterns respectively.

Fig. 3: (color online) The variation of (a) $R_{1}$ and $R_{2}$, (b) $\mathrm{Mn}-\mathrm{Sr} / \mathrm{La}$ and (c) Mn-Mn bond lengths as a function of temperature obtained from EXAFS fitting. The Debye-Waller factors corresponding to (d) $R_{1}$, (e) Sr/La, and (f) $R_{2}$ and $\mathrm{Mn}$ as a function of temperature. 

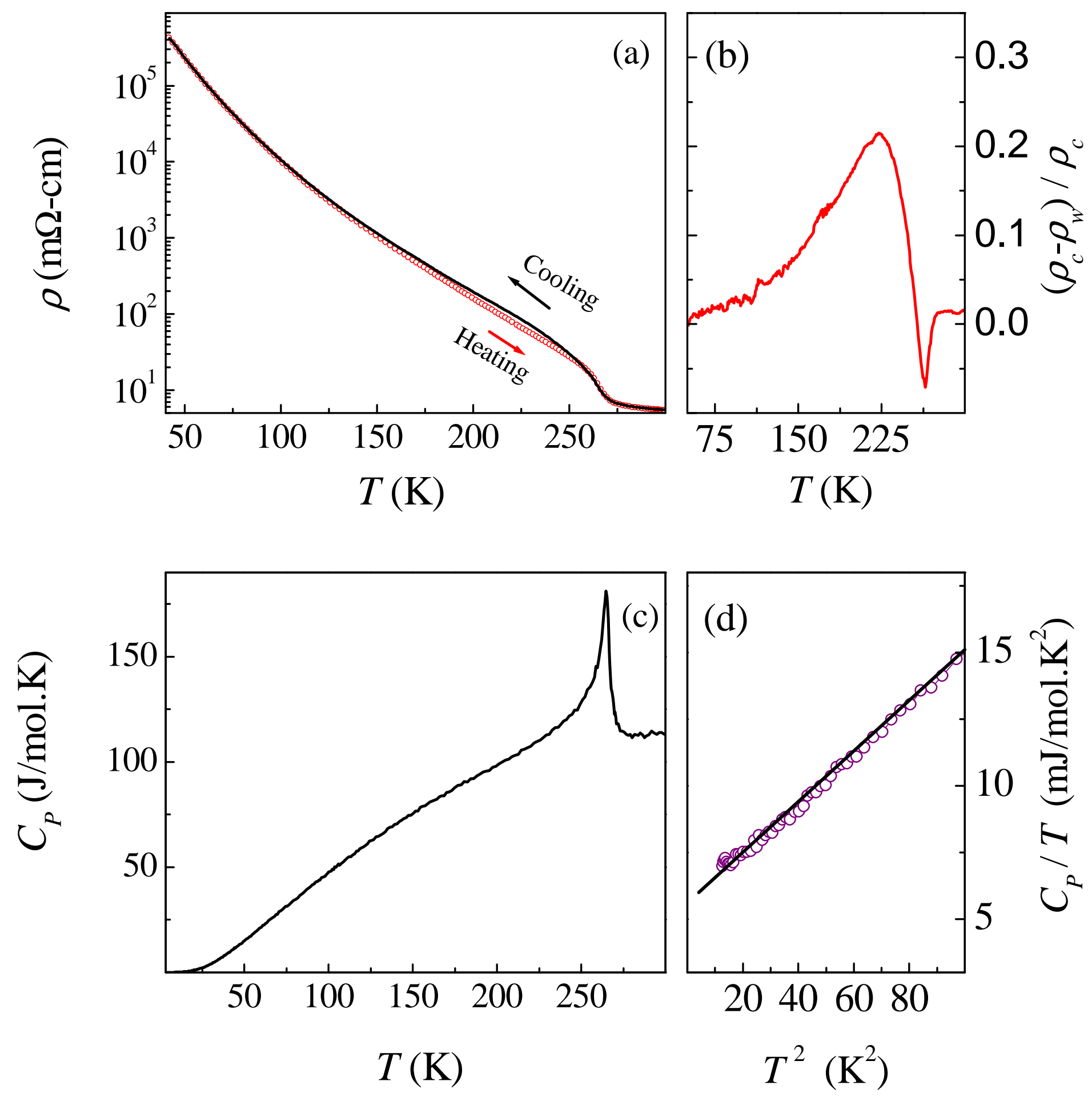


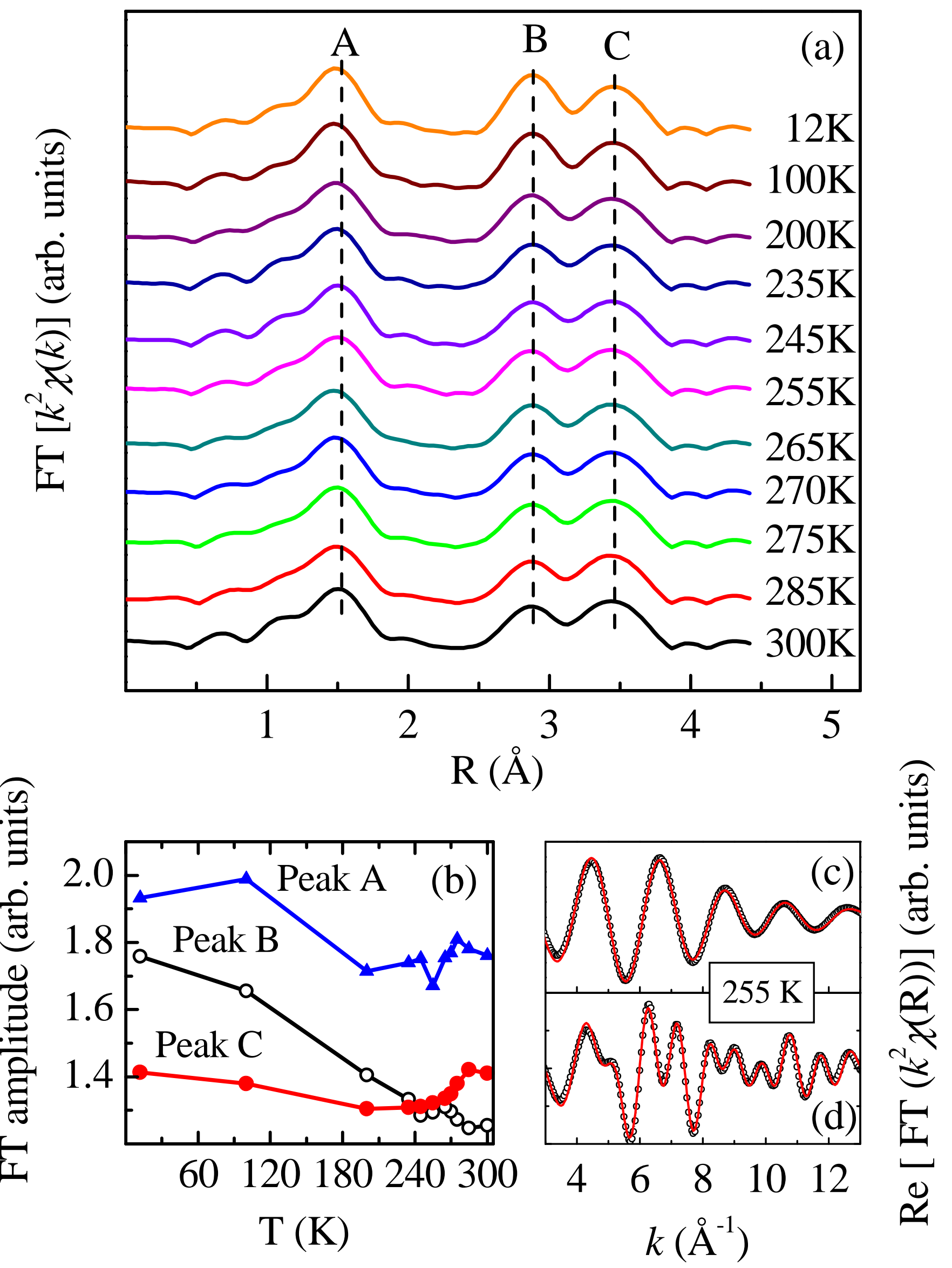




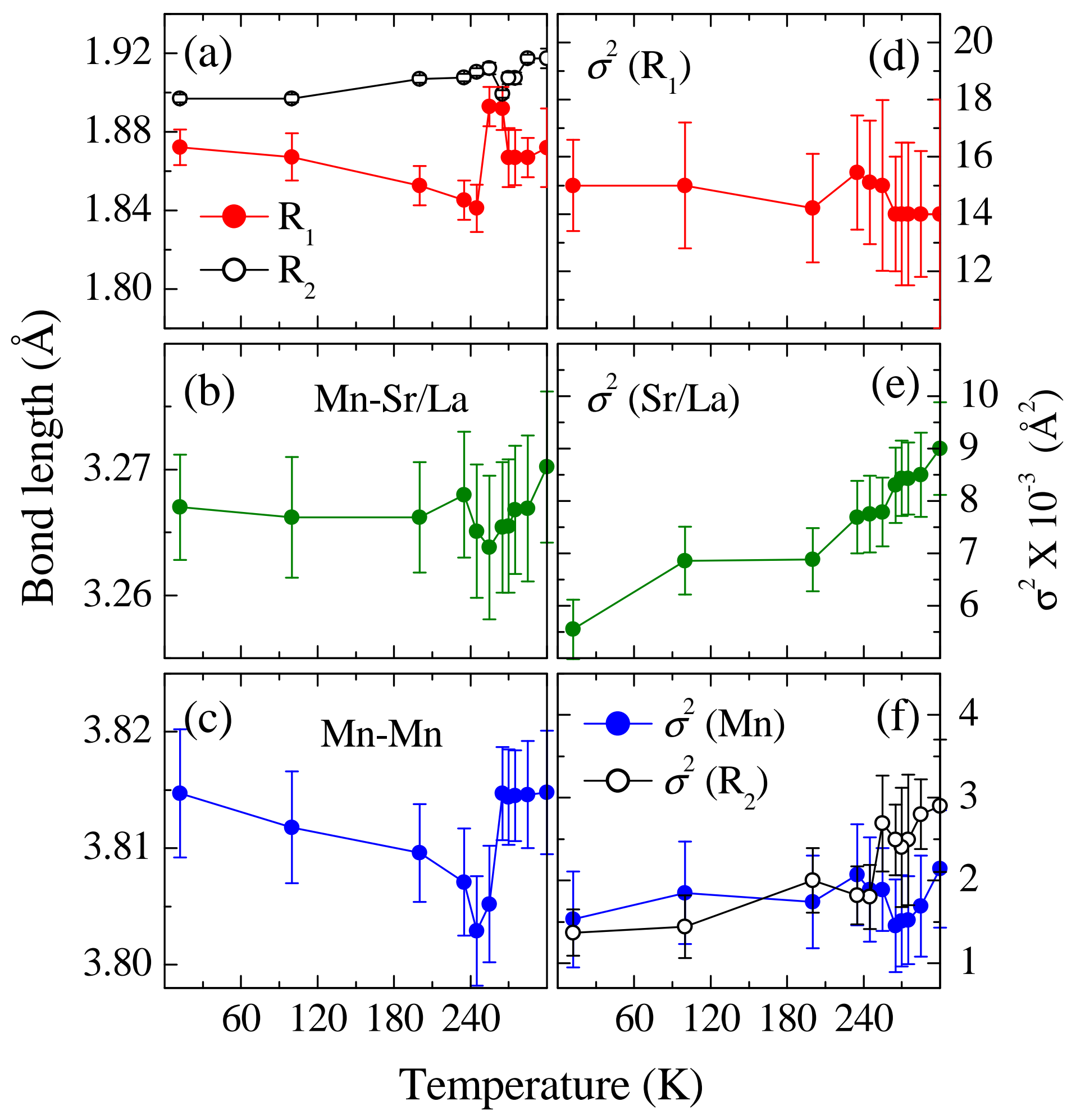

